\title{
SAE1 Gene
}

National Cancer Institute

\section{Source}

National Cancer Institute. SAE1 Gene. NCI Thesaurus. Code C101571.

This gene is involved in post-translational protein modification. 